\title{
СПЕЦИФИКА БЮДЖЕТИРОВАНИЯ
} КАК МОДЕЛИ ФИНАНСОВОГО ПЛАНИРОВАНИЯ В ХОЗЯЙСТВЕННОЙ ДЕЯТЕЛЬНОСТИ ОРГАНИЗАЦИИ

\author{
Р. Н. Жабина \\ магистрант экономического факультета, \\ Саратовский государственный университет \\ E-mail: regina-zhabina@rambler.ru
}

Введение. Бюджетирование как модель финансового планирования позволяет обосновать оптимальные уровни расхода финансовых средств предприятия и получить определенные конкурентные преимущества, что важно в условиях современного рынка. В работе выделены элементы системы финансового планирования и рассмотрены особенности бюджетирования как модели финансового планирования в хозяйственной деятельности организации, детальный подход к которым может повысить эффективность предприятия. Теоретический анализ. Основными факторами, влияющими на бюджетирование организации, названы внешние условия, в которых действует предприятие, их устойчивость или изменчивость, специфика финансовой структуры организации, принятых вертикальных взаимодействий и зависимость процесса бюджетирования от применяемых подходов, конъюнктуры рынка и организации финансового планирования на предприятии, степени детализации планов. Заключение. На основании рассмотрения специфических аспектов бюджетирования сформулированы основные направления положительного воздействия и негативного влияния на модель финансового планирования в хозяйственной деятельности организации.

Ключевые слова: бюджет, бюджетирование, финансовое планирование.

\section{Введение}

В современных рыночных условиях прогнозирование рынка - это точка отсчета любого планирования предпринимательской деятельности коммерческих структур. Каждая организация как субъект экономических взаимодействий ставит перед собой цели и задачи, рассчитанные как на краткосрочную, так и на долгосрочную перспективу. Основным методом решения всех поставленных задач является разработка планов по достижению целей. Планирование можно определить как совокупность таких факторов, как постановка цели, определение того, какие ресурсы могут быть необходимы для достижения этой цели и будут ли эти ресурсы доступны, когда в них возникнет потребность.

По содержанию различных плановых решений принято три основных уровня планирования: стратегический, тактический оперативный, которые охватывают текущую деятельность предприятия. Все это может осуществляться в форме бюджетирования. Именно бюджетирование как модель финансового планирования по- зволяет обосновать оптимальные уровни расхода финансовых средств предприятия и получить определенные конкурентные преимущества, что важно в условиях современного рынка.

Цель данной работы - выделить элементы системы финансового планирования и рассмотреть особенности бюджетирования как модели финансового планирования в хозяйственной деятельности организации, детальный подход к которым может повысить эффективность предприятия.

\section{Теоретический анализ}

Бюджет - это документ, содержащий плановые показатели организации на ближайшую перспективу [1]. Более развернутое определение бюджета дает Е. Ю. Добровольский. Бюджет предприятия - это финансовый план или выраженное в цифрах запланированное на будущее финансовое состояние предприятия или фирмы, финансовое, количественно определенное выражение результатов маркетинговых исследований и производственных планов, необходимых для достижения поставленных целей. Соответственно бюджетирование - это процесс разработки, исполнения, контроля и анализа финансового плана, охватывающего все стороны деятельности организации, позволяющий сопоставить все понесенные затраты и полученные результаты в финансовых терминах на предстоящий период в целом и по отдельным подпериодам [2].

Одной из определяющих характеристик бюджета, по мнению О. С. Красовой, является формализация, т. е. количественное выражение. Бюджет - это прежде всего набор цифр, в то время как план включает в себя помимо этого перечень конкретных мероприятий по достижению целей организации [3].

Количественное выражение является основной характеристикой бюджета, так как в большинстве случаев бюджет - это финансовый документ, но даже если предмет планирования - материальные или человеческие ресурсы, то в бюджете это отражается в количественных показателях. 
Бюджетирование - это процесс разработки, рассмотрения, утверждения и исполнения с контролем этих этапов операционных, финансовых, инвестиционных бюджетов центрами ответственности внутри отдельных подразделений организации.

Бюджетирование в общем виде можно определить как процесс составления финансовых планов, но кроме того, что бюджетирование это основной метод планирования, оно также является инструментом контроля и управления ресурсами предприятия.

На предприятии бюджетирование - это основа планирования, и главное его назначение состоит в том, что оно способствует принятию правильных управленческих решений, позволяет дать оценку всем аспектам финансовой состоятельности предприятия, помогает осуществлять контроль и управление материальными, денежными и иными ресурсами предприятия, а также работает на укрепление финансовой дисциплины. Иерархия бюджетов способствует подчинению интересов отдельных структурных подразделений интересам предприятия в целом. В конце отчетного периода проводится анализ результатов деятельности предприятия на предмет оправданности финансовых затрат на достижение поставленных целей

Исходя из вышеперечисленного, можно определить бюджетирование как производственно-финансовое планирование деятельности предприятия, осуществляемое путем составления бюджетов предприятия и его подразделений с целью определения их финансовых затрат при достижении финансовых результатов.

Стоит отметить, что некоторые авторы определяют бюджетирование не просто как процесс, а как технологию. Так, например, по мнению М. Вахрушиной, бюджетирование - это, с одной стороны, процесс составления финансовых планов и смет, а с другой, управленческая технология, предназначенная для выработки и повышения финансовой обоснованности принимаемых управленческих решений [4]. М. Горбачева также определяет бюджетирование как технологию. Бюджетирование - это технология финансового планирования, учета и контроля доходов и расходов, получаемых от бизнеса на всех уровнях управления, которая позволяет анализировать прогнозируемые и полученные финансовые показатели [5].

Бюджетирование как модель финансового планирования естественным образом зависит от объекта приложения, и говорить об особенностях бюджетирования можно лишь в каждом частном случае, зная цели, средства и инструменты, которые использует предприятие.
Далее нужно определить финансовые и нефинансовые, внутренние и внешние, субъективные и объективные, прямые и косвенные факторы, от которых зависят условия финансирования, а значит, и финансовое бюджетирование, так как финансирование - это главная операция для организации финансового планирования по модели бюджетирования.

Выделим основные факторы, влияющие на бюджетирование организации.

Прежде всего, большое значение имеют внешние условия, в которых действует предприятие, их устойчивость или изменчивость. $\mathrm{C}$ целью учета изменения внешних условий бюджетирование, кроме основной своей функции - финансового планирования, выполняет аналитическую функцию. Это позволяет обеспечить стратегическую гибкость и оперативность в процессах корректировки инструментария или же выработке альтернативных сценариев.

Не менее важное значение имеет специфика финансовой структуры организации. Говоря о структурных подразделениях предприятия, необходимо отметить их особую роль в системе бюджетирования. Каждое подразделение предприятия осуществляет расходы, планирует свои действия, приносит доходы, т.е. вносит свой вклад в конечный финансовый результат. При этом каждое подразделение несет ответственность за свои действия: осуществляет, планирует и отчитывается по результатам. Таким образом, руководство делегирует ответственность подразделениям предприятия, что является фундаментальным звеном в построении процесса бюджетирования. Более того, выделение центров финансовой ответственности можно считать первым шагом на пути создания системы бюджетирования, поскольку из таких центров формируется финансовая структура предприятия в целом.

Под центрами ответственности в управленческом учете понимаются область, сфера, вид деятельности, сегмент организации, во главе которых находится ответственное лицо (менеджер, руководитель), наделенный персональной, личной ответственностью за результаты и затраты деятельности сегмента, в рамках которого он способен оказывать на них непосредственное управленческое воздействие в пределах предоставляемых ему полномочий [6].

Видимо, в определении, данном П. М. Мансуровым, область, сферу, вид деятельности следует понимать именно как сегмент организации, поскольку, на наш взгляд, сама по себе область деятельности, даже возглавляемая ответственным лицом, не обязательно будет являться центром ответственности. Отметим, что под центром ответственности следует понимать именно струк- 
турное подразделение как сегмент организации, возглавляемый лицами, наделенными финансовой ответственностью и управленческими полномочиями.

В процессе формирования центров финансовой ответственности выстраивается их иерархия, а также определяются полномочия их руководителей и порядок расчетов финансовых результатов их деятельности. Таким образом, формируется финансовая структура организации.

Еще одной особенностью является специфика принятых вертикальных взаимодействий и зависимость процесса бюджетирования от подхода. Существует три основных подхода к формированию бюджета, основанных на специфике взаимодействия и координации между различными уровнями управления предприятием: «сверху вниз», когда бюджеты разрабатываются службами аппарата управления, а ориентирами служат целевые показатели, установленные руководством организации; «снизу вверх», когда бюджеты разрабатываются руководством структурных подразделений и корректируются службами аппарата управления организации; «встречное» формирование как некоторый синтез первых двух видов.

Теоретически на любом предприятии реализуем любой из этих трех подходов, но в каждом конкретном случае необходимо рассчитать и определить наиболее оптимальный вариант. Так, считается, что подход «снизу вверх/сверху вниз» является наиболее сбалансированным. Это отчасти может быть оправданно на крупных предприятиях с множеством подразделений, но будет менее эффективно на небольших предприятиях с несложной структурой. А при хорошо отлаженной системе учета и контроля и на крупных предприятиях, например, второй вариант «снизу вверх» может оказаться самым приемлемым в силу наибольшей простоты, так как искать баланс чаще всего более затратно, чем обеспечить точный расчет и придерживаться выработанного плана.

Следующая специфическая особенность бюджетирования связана с зависимостью от конъюнктуры рынка и с организацией финансового планирования на предприятии, и, в частности, с бюджетным периодом. Обычно бюджет предприятия разрабатывается на один календарный год, и за редким исключением такой бюджетный период можно взять за основу в процессе планирования практически любого предприятия или организации.

Наибольший интерес для нас представляют подпериоды, на которые можно разбить бюджетный период. Это связано с тем, что на любом рынке товаров и услуг существуют сезонные колебания, сопровождаемые изменением конъюнктуры потребления. Например, для промышленного предприятия, производящего станки и оборудование, это не имеет особого значения, и бюджетный период там разбивается в основном на кварталы и месяцы. В этом случае в расчет берутся усредненные показатели, и стратегия планирования разрабатывается на основе анализа исполнения годового бюджета в целом.

Но существуют предприятия, деятельность которых напрямую связана с изменением сезонных колебаний, и в этом случае наиболее целесообразной может оказаться разбивка на подпериоды, максимально привязанные к изменениям конъюнктуры рынка. Такая привязка может помочь наиболее эффективно распределить движение товарно-материальных и финансовых потоков.

Степень детализации планов также является специфической чертой бюджетирования. Критерии достаточности и уместности детализации планов можно сформулировать следующим образом. Степень детализации планов должна быть такой, чтобы на основе некоторого плана можно было подготовить другие планы, следующие за ним в иерархии планирования. Будущий пользователь плана должен иметь возможность почерпнуть из него всю необходимую управленческую информацию. Степень детализации планов необходимо оценивать по трем направлениям: детализация с точки зрения количества содержащихся в каждом плане показателей, т.е. какого рода информацию можно вообще почерпнуть из плана; детализация каждого показателя, т.е. выделены ли внутри отдельных показателей какие-либо подстатьи, подпункты и т.д.; детализация горизонта планирования, т.е. выделены ли внутри периода планирования какие-либо дополнительные временные отрезки.

\section{Заключение}

На основании рассмотренных специфических аспектов бюджетирования возможно сформулировать основные направления влияния бюджетирования на модель финансового планирования в хозяйственной деятельности организации.

Так, положительное воздействие бюджетирования на мотивацию и настрой коллектива обусловлено вовлечением в процедуру составления планов широкого круга подразделений и их сотрудников.

Примером этого является ситуация, когда на предприятии имеется производственный цех, который относится к центру затрат, поскольку не имеет выхода на рынок и, следовательно, не имеет доходов. Мы можем начать условно оценивать произведенную продукцию по рыноч- 
ной цене, и у данного подразделения появится условный доход. Таким образом, для подобного подразделения мы можем составить «Бюджет доходов и расходов» с целью оценки деятельности подразделения и повышения мотивации его сотрудников.

Бюджетирование усиливает координацию работы предприятия в целом. Наличие среди перечня используемых планов взаимосвязанных и согласованных между собой операционных планов, таких как план продаж, план производства и план потребности в ресурсах, предусматривает согласованность всех звеньев системы управления, что, в свою очередь, обеспечивает координацию деятельности предприятия в целом.

Процедура выявления и анализа отклонений между фактическими и плановыми значениями, а также определения причин и «виновников» отклонений позволяет разработать систему мероприятий по предотвращению неблагоприятных отклонений в будущем. Наличие и согласованность между собой плана потребности в материалах, плана закупок материалов и плана потребности в персонале позволяет усовершенствовать процесс распределения ресурсов.

Введение процедур контроля за исполнением бюджета по итогам периода, а также наличие таких параметров бюджета, как «фактические показатели» и «отклонения фактических и плановых показателей», обеспечивает возможность оперативной корректировки деятельности организации.

Бюджетирование также повышает «финансовую прозрачность» для собственников, акционеров и других заинтересованных лиц, что, в свою очередь, способствует улучшению инвестиционного климата.

Однако бюджетирование имеет в некоторых аспектах хозяйственной деятельности организации и негативное влияние. Например, различное восприятие бюджетов разными людьми. Ведь бюджеты не всегда способны помочь в решении повседневных, текущих проблем, не всегда отражают причины событий и отклонений, не всегда учитывают изменения условий, кроме того, не все менеджеры обладают достаточной подготовкой для анализа финансовой информации.

Системы бюджетирования обладают свойствами сложности и дороговизной. Если бюджеты не доведены до сведения каждого сотрудника, то они не оказывают практически никакого влияния на мотивацию и результаты работы, а вместо этого воспринимаются исключительно как средство для оценки деятельности работников и отслеживания ошибок.

Бюджеты требуют от сотрудников высокой производительности труда; в свою очередь, сотрудники противодействуют этому, стараясь минимизировать свою нагрузку, что приводит к конфликтам, вызывает состояние подавленности, страха, а следовательно, снижает эффективность работы.

Бюджетирование обладает свойством противоречия между достижимостью целей и их стимулирующим эффектом: если достичь поставленных целей слишком легко, то бюджет не имеет стимулирующего эффекта для повышения производительности; если слишком сложно стимулирующий эффект пропадает, поскольку никто не верит в возможность достижения целей [7].

С нашей точки зрения, существует подход, который позволит найти оптимальный баланс между мотивацией сотрудников и выполнением бюджетных критериев. В частности, разрабатывая систему стимулирования, необходимо точно определить факторы, оказавшие непосредственное влияние на бюджетные показатели. С учетом этого появится возможность материально стимулировать именно непосредственных исполнителей. При этом руководителей, ответственных за разработку и выполнение бюджета, следует поощрять за планирование и прогнозирование, не влекущее за собой неблагоприятных отклонений. Это позволит сформировать бюджет, выполнимый по всем параметрам, поскольку такой бюджет не будет содержать «напряженных» и «неадекватных» показателей.

Таким образом, бюджетирование как модель финансового планирования - эффективный инструмент достижения стратегических целей организации, основной из которых является улучшение финансовых показателей.

\section{Список литературы}

1. Лаврушин О. И. Бюджетирование : кратко о главном. URL: www.elitarium.ru (дата обращения: 12.12. 2013).

2. Добровольский Е. Ю., Карабанов Б. М., Боровков П. С., Глухов Е. В., Бреслав Е. П. Бюджетирование : шаг за шагом. СПб. : Питер, 2005. 448 с.

3. Красова О. С. Бюджетирование и контроль затрат : теория и практика. М. : Омега-Л, 2008. 264 с.

4. Вахрушина М. Бюджетирование и управленческий контроль // Экономика и жизнь (приложение). 2001. № 45. C. 12-13.

5. Горбачева Л. Постановка системы бюджетирования // Финансовая газета. 2002. № 20. С. 11-15.

6. Мансуров П. М. Управленческий учет : учеб. пособие. Ульяновск : УлГТУ, 2010. 175 с.

7. Кучеренко А. И. Бюджетирование как метод финансового планирования деятельности организации // Справочник экономиста. 2010. № 3. С. 34-43. 


\section{Specifics of Budgeting as a Model of Financial Planning in Organization Economic Activities}

\section{R. N. Zabina}

Saratov State University

83, Astrakhanskaya, Saratov, 410012, Russia

E-mail: regina-zhabina@rambler.ru

Introduction. Budgeting as a financial planning model allows to prove the optimal consumption levels of funding and allows enterprises to obtain competitive advantages, which is important in today's market. The paper outlines the elements of the financial planning and budgeting as the features of the model financial planning activities of the organization, a detailed approach which can improve the efficiency of the company. Theoretical analysis. The main factors influencing the budgeting organization, highlighted the external environment in which the company operates, their stability or volatility, the specific financial structure of the organization adopted vertical interactions and the dependence of the budgeting process from the existing approaches, the market conditions and the organization of financial planning at the enterprise level of detail plans. Conclusion. Based on the review of specific aspects of budgeting the main directions of the positive effects and the negative impact on the financial planning model in the activities of the organization.

Key words: budget, budgeting, financial planning.

\section{References}

1. Lavrushin O. I. Biudzhetirovanie: kratko o glavnom (Budgeting: briefly about the main thing). Available at: www.elitarium.ru (accessed 12 December 2013).

2. Dobrovolsky E. Yu., Karabanov B. M., Borovkov P. S., Glukhov E. V., Breslav E. P. Biudzhetirovanie: shag za shagom [Budgeting: step by step]. St.-Petersburg, Piter Publ., 2005. 448 p.

3. Krasova O. S. Biudzhetirovanie i kontrol'zatrat: teoriia i praktika [Budgeting and Cost Control: Theory and Practice]. Moscow, Omega-L Publ., 2008. 264 p.

4. Vakhrushina M. Biudzhetirovanie i upravlencheskii kontrol' [Budgeting and management control]. Ekonomika i zhizn'(prilozhenie) [Economy and Life (application)], 2001, no. 45, pp. 12-13.

5. Gorbacheva L. Postanovka sistemy biudzhetirovaniia [Organization of budgeting system]. Finansovaia gazeta [Financial newspaper], 2002, no. 20, pp. 11-15.

6. Mansurov P. M. Upravlencheskii uchet: uchebnoe posobie [Managerial Accounting: Tutorial]. Ul'ianovsk, Ul'ianovsk State Technical University Publ., 2010. $175 \mathrm{p}$.

7. Kucherenko A. I. Biudzhetirovanie kak metod finansovogo planirovaniia deiatel'nosti organizatsii [Budgeting as a method of financial planning organization]. Spravochnik ekonomista [Directory economist], 2010, no. 3, pp. 34-43. 